\title{
The Generalized Well-Posedness of the Cauchy Problem for an Abstract Stochastic Equation with Multiplicative Noise
}

\author{
I. V. Melnikova ${ }^{1}$ and M. A. Alshanskiy ${ }^{2}$ \\ Received May 23, 2011
}

\begin{abstract}
We study the existence, uniqueness, and stability of a solution to the Cauchy problem for a stochastic differential equation with multiplicative noise in the spaces of generalized random variables with values in a Hilbert space.

Keywords: Cauchy problem, semigroup, white noise, generalized solutions.
\end{abstract}

DOI:10.1134/S0081543813020119

\section{INTRODUCTION}

The desire to take into account the influence of random factors on a system in the construction of its mathematical model in the form of a differential equation results in stochastic differential equations. In many applied problems, for example, in problems of population dynamics and in problems of financial mathematics concerned with the calculation of interest rates and current bond prices, stochastic differential equations are considered in infinite-dimensional spaces.

The introduction of white noise into an equation is related to the problem of a correct definition of a random process such that its values at different times are independent random variables with infinite deviations. Thus, as early as at the stage of the formulation of stochastic problems with randomness of the white noise type, the problem of divergence elimination arises. This problem remains important at the stage of the construction of solutions, since the presence of a term with noise in the equation naturally worsens the properties of solutions as compared to the deterministic case. One of the possible approaches to resolving this difficulty is the formulation and solution of the problem in appropriate spaces of generalized functions.

Consider the classical formulation (in the form of Ito differentials) of the Cauchy problem for a linear equation with multiplicative noise:

$$
d X(t)=A X(t) d t+B(X(t)) d W(t), \quad t \geq 0, \quad X(0)=\Phi,
$$

where $A: H \rightarrow H$ and $B(\cdot): H \rightarrow \mathcal{L}(H)$ are linear operators ( $H$ is a separable Hilbert space), $\Phi$ is an $H$-valued random variable, and $W(t)$ is an $H$-valued cylindrical Wiener process. In fact,

\footnotetext{
${ }^{1}$ Institute of Mathematics and Computer Science, Ural Federal University, pr. Lenina 51, Yekaterinburg, 620000 Russia email: Irina.Melnikova@usu.ru

${ }^{2}$ Institute of Radioelectronics and Informational Technologies, Ural Federal University, ul. Mira 19, Yekaterinburg, 620002 Russia

email: mxalsh@gmail.com
} 
equation (1.1) is an "integrated" differential equation written in the form of differentials. Because of the nonsmoothness of white noise, the passage to this equation is implemented by means of the Ito integral technique. As a result, the existence of the so-called "soft" solution (see, for example, [1]) can be proved for an operator $A$ generating a semigroup of class $C_{0}$.

In the present paper, we introduce spaces of generalized $H$-valued random variables; in these spaces, $H$-valued white noise is smooth in the variable $t$. (Spaces of $\mathbb{R}$-valued generalized random variables are described in $[2,3]$ and other papers.) This makes it possible to consider the Cauchy problem for the differential equation

$$
\frac{d X(t)}{d t}=A X(t)+B(X(t)) \diamond \mathbb{W}(t), \quad t \geq 0, \quad X(0)=\Phi,
$$

where $\mathbb{W}(t)$ is white noise and " $\diamond$ " denotes the Wick product of generalized random variables.

The existence of a unique solution of (1.2) is proved by reducing it to a deterministic Cauchy problem for a differential operator equation in a Hilbert space by means of an $S$-transformation. Estimates for the solution are obtained, which can be interpreted as a certain stability with respect to a variation of the initial data. Thus, the generalized well-posedness of problem (1.2) is proved.

\section{GENERALIZED RANDOM VARIABLES}

Let $\mathcal{S}^{\prime}$ be the space of slow-growth distributions over the space of rapidly decreasing functions $\mathcal{S}$. The space $\mathcal{S}$ is a countably Hilbert kernel space: $\mathcal{S}=\cap_{p \in \mathbb{N}} \mathcal{S}_{p}$, where $\mathcal{S}_{p}$ is a subspace of $L^{2}(\mathbb{R})$ with the norm $|\cdot|_{p}$ defined by the scalar product $(f, g)_{p}:=\left(\hat{D}^{p} f, \hat{D}^{p} g\right)_{L^{2}(\mathbb{R})}$, where

$$
\hat{D}=-\frac{d^{2}}{d x^{2}}+x^{2}+1
$$

Let $\mathcal{B}\left(\mathcal{S}^{\prime}\right)$ be the $\sigma$-algebra of Borel subsets of $\mathcal{S}^{\prime}$, and let $\mu$ be the probability measure on $\mathcal{B}\left(\mathcal{S}^{\prime}\right)$ (the Minlos-Sazonov measure) satisfying the condition

$$
\int_{\mathcal{S}^{\prime}} e^{i\langle\omega, \theta\rangle} d \mu(\omega)=e^{-\frac{1}{2}|\theta|_{0}^{2}}, \quad \theta \in \mathcal{S},
$$

where $|\cdot|_{0}$ is the norm of $L_{2}(\mathbb{R})$. The measure $\mu$ is called the normalized Gaussian measure on $\mathcal{S}^{\prime}$, since, for any set of functions $\theta_{1}, \theta_{2}, \ldots, \theta_{n} \in \mathcal{S}$ orthogonal in $L^{2}(\mathbb{R})$, the random variable $\omega \mapsto\left(\left\langle\omega, \theta_{1}\right\rangle,\left\langle\omega, \theta_{2}\right\rangle, \ldots,\left\langle\omega, \theta_{n}\right\rangle\right)$ has normal distribution with density

$$
\frac{1}{(2 \pi)^{\frac{n}{2}} \prod_{i=1}^{n}\left|\theta_{i}\right|_{0}} \exp \left(-\frac{1}{2} \sum_{i=1}^{n} \frac{x_{i}^{2}}{\left|\theta_{i}\right|_{0}^{2}}\right)
$$

which is equivalent to the equality

$$
E\left(f\left(\left\langle\omega, \theta_{1}\right\rangle, \ldots,\left\langle\omega, \theta_{n}\right\rangle\right)\right)=\frac{1}{(2 \pi)^{\frac{n}{2}} \prod_{i=1}^{n}\left|\theta_{i}\right|_{0}} \int_{\mathbb{R}^{n}} f\left(x_{1}, \ldots, x_{n}\right) e^{-\frac{1}{2} \sum_{i=1}^{n} \frac{x_{i}^{2}}{\left|\theta_{i}\right|_{0}^{2}}} d x_{1} \ldots d x_{n}
$$

for any function $f$ such that the integral on the right-hand side exists.

The probability space $\left(\mathcal{S}^{\prime}, \mathcal{B}\left(\mathcal{S}^{\prime}\right), \mu\right)$ is called the white noise space. Denote by $\left(L^{2}\right)$ the space $L^{2}\left(\mathcal{S}^{\prime}, \mu ; \mathbb{R}\right)$ of functions (random variables) with values in $\mathbb{R}$ that are defined on $\mathcal{S}^{\prime}$ and square 
integrable with respect to the measure $\mu$, and denote the norm of this space by $\|\cdot\|_{0}$. As follows from (2.2), the equalities

$$
(\langle\cdot, \theta\rangle,\langle\cdot, \eta\rangle)_{\left(L^{2}\right)}=E(\langle\cdot, \theta\rangle\langle\cdot, \eta\rangle)=(\theta, \eta)_{L^{2}(\mathbb{R})}, \quad\|\langle\cdot, \theta\rangle\|_{0}^{2}=E\langle\cdot, \theta\rangle^{2}=|\theta|_{0}^{2}
$$

hold for any $\theta, \eta \in \mathcal{S}$. Therefore, we can extend the mapping $\theta \mapsto\langle\cdot, \theta\rangle$ by continuity from $\mathcal{S}$ to the whole space $L^{2}(\mathbb{R})$, preserving the same notation $\langle\cdot, \phi\rangle \in\left(L^{2}\right)$ in the case $\phi \in L^{2}(\mathbb{R})$. Relation (2.1) remains valid for $\theta \in L^{2}(\mathbb{R})$, and equality $(2.2)$ is true for $\theta_{1}, \ldots, \theta_{n} \in L^{2}(\mathbb{R})$.

2.1. Spaces of $\mathbb{R}$-valued generalized random variables. Let $\left\{\xi_{k}\right\}_{k=1}^{\infty}$ be the orthonormal basis of the space $L^{2}(\mathbb{R})$ consisting of the Hermite functions

$$
\xi_{k}(x)=\pi^{-\frac{1}{4}}((k-1) !)^{-\frac{1}{2}} e^{-\frac{x^{2}}{2}} h_{k-1}(x),
$$

where $\left\{h_{k}(x)\right\}_{k=0}^{\infty}$ are the Hermite polynomials $h_{k}(x)=(-1)^{n} e^{\frac{x^{2}}{2}}(d / d x)^{k} e^{-\frac{x^{2}}{2}}$, which are eigenfunctions of the differential operator $\hat{D}: \hat{D} \xi_{i}=(2 i) \xi_{i}, i \in \mathbb{N}$. Let $\mathcal{T} \subset(\mathbb{N} \cup\{0\})^{\mathbb{N}}$ be the set of all finite multiindices. The stochastic Hermite polynomials defined by the equality $\mathbf{h}_{\alpha}(\omega):=$ $\prod_{k} h_{\alpha_{k}}\left(\left\langle\omega, \xi_{k}\right\rangle\right), \omega \in \mathcal{S}^{\prime}, \alpha \in \mathcal{T}$, form an orthogonal basis of the space $\left(L^{2}\right)$; moreover,

$$
\left(\mathbf{h}_{\alpha}, \mathbf{h}_{\beta}\right)_{\left(L^{2}\right)}=\left\{\begin{array}{ll}
0, & \alpha \neq \beta, \\
\alpha !, & \alpha=\beta,
\end{array} \quad \alpha !:=\prod_{k} \alpha_{k} !\right.
$$

The Gelfand triple

$$
(\mathcal{S})_{\rho} \subset\left(L^{2}\right) \subset(\mathcal{S})_{-\rho} \quad(0 \leq \rho \leq 1)
$$

is used in $[2,3]$ to equip the Hilbert space $\left(L^{2}\right)$. The space $(\mathcal{S})_{\rho}$ plays the role of a space of test functions (random variables). By definition, $(\mathcal{S})_{\rho}=\cap_{p \in \mathbb{N}}\left(\mathcal{S}_{p}\right)_{\rho}$ with projective limit topology, where

$$
\left(\mathcal{S}_{p}\right)_{\rho}=\left\{\varphi=\sum_{\alpha \in \mathcal{T}} \varphi_{\alpha} \mathbf{h}_{\alpha} \in\left(L^{2}\right): \sum_{\alpha \in \mathcal{T}}(\alpha !)^{1+\rho}\left|\varphi_{\alpha}\right|^{2}(2 \mathbb{N})^{2 p \alpha}<\infty\right\}
$$

with the norm $|\cdot|_{p, \rho}$ generated by the scalar product

$$
(\varphi, \psi)_{p, \rho}=\sum_{\alpha \in \mathcal{T}}(\alpha !)^{1+\rho} \varphi_{\alpha} \bar{\psi}_{\alpha}(2 \mathbb{N})^{2 p \alpha}, \quad(2 \mathbb{N})^{p \alpha}:=\prod_{i \in \mathbb{N}}(2 i)^{p \alpha_{i}}
$$

The space $(\mathcal{S})_{-\rho}$ plays the role of a space of generalized functions (random variables). By definition, $(\mathcal{S})_{-\rho}=\cup_{p \in \mathbb{N}}\left(\mathcal{S}_{-p}\right)_{-\rho}$ with inductive limit topology, where $\left(\mathcal{S}_{-p}\right)_{-\rho}$ is the space conjugate to $\left(\mathcal{S}_{p}\right)_{\rho}$. The space $\left(\mathcal{S}_{-p}\right)_{-\rho}$ can be identified with the Hilbert space of all possible formal expansions $\Phi=$ $\sum_{\alpha \in \mathcal{T}} \Phi_{\alpha} \mathbf{h}_{\alpha}$ satisfying the condition

$$
\sum_{\alpha \in \mathcal{T}}(\alpha !)^{1-\rho}\left|\Phi_{\alpha}\right|^{2}(2 \mathbb{N})^{-2 p \alpha}<\infty
$$

with the scalar product

$$
(\Phi, \Psi)_{-p,-\rho}=\sum_{\alpha \in \mathcal{T}}(\alpha !)^{1-\rho} \Phi_{\alpha} \bar{\Psi}_{\alpha}(2 \mathbb{N})^{-2 p \alpha}
$$


The norm of the space $\left(\mathcal{S}_{-p}\right)_{-\rho}$ will be denoted by $|\cdot|_{-p,-\rho}$. For

$$
\Phi=\sum_{\alpha \in \mathcal{T}} \Phi_{\alpha} \mathbf{h}_{\alpha} \in(\mathcal{S})_{-\rho}, \quad \varphi=\sum_{\alpha \in \mathcal{T}} \varphi_{\alpha} \mathbf{h}_{\alpha} \in(\mathcal{S})_{\rho}
$$

we have

$$
\langle\Phi, \varphi\rangle=\sum_{\alpha \in \mathcal{T}} \alpha ! \Phi_{\alpha} \bar{\varphi}_{\alpha} .
$$

A set $M \subseteq(\mathcal{S})_{\rho}$ is called bounded if, for any sequence $\left\{\varphi_{n}\right\} \subseteq M$ and any sequence $\left\{\varepsilon_{n}\right\} \subset \mathbb{R}$ convergent to zero, $\left\{\varepsilon_{n} \varphi_{n}\right\}$ converges to zero in $(\mathcal{S})_{\rho}$. Evidently, the boundedness of a set in $(\mathcal{S})_{\rho}$ is equivalent to its boundedness in each space $\left(\mathcal{S}_{p}\right)_{\rho}$.

2.2. Spaces of $H$-valued generalized random variables. Let $H$ be a separable Hilbert space over $\mathbb{C}$ with scalar product $(\cdot, \cdot)$ and corresponding norm $\|\cdot\|$. Denote by $\left(L^{2}\right)(H)$ the space of $H$-valued functions that are defined on $\mathcal{S}^{\prime}$ and Bochner square integrable with respect to the measure $\mu$. Let $\left\{e_{j}\right\}_{j=1}^{\infty}$ be an orthonormal basis in the space $H$. The family of $H$-valued functions of the form $\left\{\mathbf{h}_{\alpha} e_{j}\right\}_{\alpha \in \mathcal{T}, j \in \mathbb{N}}$ forms an orthogonal basis of $\left(L^{2}\right)(H)$. Elements $f \in\left(L^{2}\right)(H)$ are expanded in Fourier series in this basis as follows [4-6]:

$$
\begin{gathered}
f=\sum_{\alpha \in \mathcal{T}, j \in \mathbb{N}} f_{\alpha, j} \mathbf{h}_{\alpha} e_{j}=\sum_{\alpha \in \mathcal{T}} f_{\alpha} \mathbf{h}_{\alpha}=\sum_{j=1}^{\infty} f_{j} e_{j}, \\
f_{\alpha, j} \in \mathbb{R}, \quad f_{\alpha}=\sum_{j} f_{\alpha, j} e_{j} \in H, \quad f_{j}=\sum_{\alpha \in \mathcal{T}} f_{\alpha, j} \mathbf{h}_{\alpha} \in\left(L^{2}\right) ;
\end{gathered}
$$

moreover,

$$
\|f\|_{\left(L^{2}\right)(H)}^{2}=\sum_{\alpha \in \mathcal{T}, j \in \mathbb{N}} \alpha !\left|f_{\alpha, j}\right|^{2}=\sum_{\alpha \in \mathcal{T}} \alpha !\left\|f_{\alpha}\right\|_{H}^{2}=\sum_{j=1}^{\infty}\left\|f_{j}\right\|_{\left(L^{2}\right)}^{2} .
$$

Let us define the space $(\mathcal{S})_{-\rho}(H)$ of $H$-valued generalized functions over the space of test functions $(\mathcal{S})_{\rho}$ as the space of linear continuous operators $\Phi:(\mathcal{S})_{\rho} \rightarrow H$ with topology of uniform convergence on bounded subsets of $(\mathcal{S})_{\rho}$. The action of an element $\Phi \in(\mathcal{S})_{-\rho}(H)$ on a test function $\varphi \in(\mathcal{S})_{\rho}$ will be denoted by $\Phi[\varphi]$.

Proposition 1. Any $\Phi \in(\mathcal{S})_{-\rho}(H)$ is a bounded operator from $\left(\mathcal{S}_{p}\right)_{\rho}$ to $H$ for some $p \in \mathbb{N}$.

Proof. Assume that this is not so. Let $\Phi \in(\mathcal{S})_{-\rho}(H)$. For any $p \in \mathbb{N}$, choose $\varphi_{p} \in\left(\mathcal{S}_{p}\right)_{\rho}$ so that $\left|\varphi_{p}\right|_{p, \rho}=1$ and $\left\|\Phi\left[\varphi_{p}\right]\right\| \geq p$. In view of the inequalities $\left|\varphi_{k}\right|_{p, \rho} \leq\left|\varphi_{k}\right|_{k, \rho}$, which hold for $k>p$, the sequence $\left\{\frac{\varphi_{k}}{k}\right\}$ converges to zero in the space $(\mathcal{S})_{\rho}$ and $\left\|\Phi\left[\frac{\varphi_{k}}{k}\right]\right\| \geq 1$, which contradicts the continuity of $\Phi$.

The space of test functions $(\mathcal{S})_{\rho}$ is a kernel countably Hilbert space, since, for any $p \in \mathbb{N}$, the embedding operator $I_{p, p+1}:\left(\mathcal{S}_{p+1}\right)_{\rho} \hookrightarrow\left(\mathcal{S}_{p}\right)_{\rho}$ is a Hilbert-Schmidt operator. Indeed, for the orthonormal basis of $\left(\mathcal{S}_{p+1}\right)_{\rho}$

$$
\left\{\frac{\mathbf{h}_{\alpha}}{(\alpha !)^{\frac{1+\rho}{2}}(2 \mathbb{N})^{(p+1) \alpha}}\right\}
$$

we have

$$
\sum_{\alpha \in \mathcal{T}}\left|\frac{\mathbf{h}_{\alpha}}{(\alpha !)^{\frac{1+\rho}{2}}(2 \mathbb{N})^{(p+1) \alpha}}\right|_{p, \rho}^{2}=\sum_{\alpha \in \mathcal{T}} \frac{1}{(2 \mathbb{N})^{2 \alpha}}<\infty .
$$


Define

$$
A(p)=\sum_{\alpha \in \mathcal{T}} \frac{1}{(2 \mathbb{N})^{p \alpha}} .
$$

As proved in [3], this series converges for $p>1$.

This and Proposition 1 imply the following statement.

Proposition 2. Any element $\Phi \in(\mathcal{S})_{-\rho}(H)$ is a Hilbert-Schmidt operator from $\left(\mathcal{S}_{p}\right)_{\rho}$ to $H$ for some $p \in \mathbb{N}$.

For any $\Phi \in(\mathcal{S})_{-\rho}(H)$, we denote by $\Phi_{j}$ the linear functional defined on $\varphi \in(\mathcal{S})_{\rho}$ by the equality $\left\langle\Phi_{j}, \varphi\right\rangle:=\left(\Phi[\varphi], e_{j}\right)$. Let $\Phi$ be a Hilbert-Schmidt operator from $\left(\mathcal{S}_{p}\right)_{\rho}$ to $H$. Then, all the functionals $\Phi_{j}, j \in \mathbb{N}$, belong to the corresponding conjugate space $\left(\mathcal{S}_{-p}\right)_{-\rho}$ and, hence, admit the expansion

$$
\Phi_{j}=\sum_{\alpha \in \mathcal{T}} \Phi_{\alpha, j} \mathbf{h}_{\alpha}, \quad \sum_{\alpha \in \mathcal{T}}(\alpha !)^{1-\rho}\left|\Phi_{\alpha, j}\right|^{2}(2 \mathbb{N})^{-2 p \alpha}<\infty .
$$

For the norm of $\Phi$ as a Hilbert-Schmidt operator from $\left(\mathcal{S}_{p}\right)_{\rho}$ to $H$, we have

$$
\begin{gathered}
\|\Phi\|_{\mathrm{HS}, p, \rho}^{2}=\sum_{\alpha \in \mathcal{T}}\left\|\Phi\left[\frac{\mathbf{h}_{\alpha}}{(\alpha !)^{\frac{1+\rho}{2}}(2 \mathbb{N})^{p \alpha}}\right]\right\|^{2} \\
=\sum_{\alpha \in \mathcal{T}} \sum_{j=1}^{\infty}\left|\left\langle\Phi_{j}, \frac{\mathbf{h}_{\alpha}}{(\alpha !)^{\frac{1+\rho}{2}}(2 \mathbb{N})^{p \alpha}}\right\rangle\right|^{2}=\sum_{\alpha \in \mathcal{T}, j \in \mathbb{N}}(\alpha !)^{1-\rho}\left|\Phi_{\alpha, j}\right|^{2}(2 \mathbb{N})^{-2 p \alpha} .
\end{gathered}
$$

Denote by $\operatorname{HS}\left(\left(\mathcal{S}_{p}\right)_{\rho} ; H\right)$ the space of Hilbert-Schmidt operators from $\left(\mathcal{S}_{p}\right)_{\rho}$ to $H$. This is a separable Hilbert space. The operators $\mathbf{h}_{\alpha} \otimes e_{j}, \alpha \in \mathcal{T}, j \in \mathbb{N}$, defined by the equality

$$
\left(\mathbf{h}_{\alpha} \otimes e_{j}\right) \varphi:=\left(\mathbf{h}_{\alpha}, \varphi\right)_{\left(L^{2}\right)} e_{j}, \quad \varphi \in\left(\mathcal{S}_{p}\right)_{\rho}
$$

form an orthogonal basis of this space. As follows from Proposition 2,

$$
(\mathcal{S})_{-\rho}(H)=\bigcup_{p \in \mathbb{N}} \operatorname{HS}\left(\left(\mathcal{S}_{p}\right)_{\rho} ; H\right)
$$

and any element $\Phi \in(\mathcal{S})_{-\rho}(H)$ admits the expansions

$$
\Phi[\cdot]=\sum_{j \in \mathbb{N}}\left\langle\Phi_{j}, \cdot\right\rangle e_{j}=\sum_{\alpha \in \mathcal{T}, j \in \mathbb{N}} \Phi_{\alpha, j}\left(\mathbf{h}_{\alpha} \otimes e_{j}\right)=\sum_{\alpha \in \mathcal{T}} \Phi_{\alpha}\left(\mathbf{h}_{\alpha}, \cdot\right)_{\left(L^{2}\right)},
$$

where $\Phi_{j}=\left(\Phi[\cdot], e_{j}\right) \in\left(\mathcal{S}_{-p}\right)_{-\rho}$ for some $p \in \mathbb{N}$ and $\Phi_{\alpha}=\sum_{j \in \mathbb{N}} \Phi_{\alpha, j} e_{j} \in H$. Moreover,

$$
\|\Phi\|_{\mathrm{HS}, p, \rho}^{2}=\sum_{j \in \mathbb{N}}\left|\Phi_{j}\right|_{-p,-\rho}^{2}=\sum_{\alpha \in \mathcal{T}, j \in \mathbb{N}}(\alpha !)^{1-\rho}\left|\Phi_{\alpha, j}\right|^{2}(2 \mathbb{N})^{-2 p \alpha}=\sum_{\alpha \in \mathcal{T}}(\alpha !)^{1-\rho}\left\|\Phi_{\alpha}\right\|^{2}(2 \mathbb{N})^{-2 p \alpha}<\infty
$$

Evidently,

$$
\operatorname{HS}\left(\left(\mathcal{S}_{p_{1}}\right)_{\rho} ; H\right) \subseteq \operatorname{HS}\left(\left(\mathcal{S}_{p_{2}}\right)_{\rho} ; H\right) \text { for } p_{1}<p_{2}
$$

and

$$
\|\Phi\|_{H S, p_{1}, \rho} \geq\|\Phi\|_{H S, p_{2}, \rho} \quad \text { for all } \Phi \in \operatorname{HS}\left(\left(\mathcal{S}_{p_{1}}\right)_{\rho} ; H\right) .
$$


A set $\mathcal{M} \subseteq(\mathcal{S})_{-\rho}(H)$ is called bounded if, for any sequence $\left\{\Phi_{n}\right\} \subseteq \mathcal{M}$ and any sequence $\left\{\varepsilon_{n}\right\} \subset \mathbb{R}$ convergent to zero, the sequence $\left\{\varepsilon_{n} \Phi_{n}\right\}$ converges to zero in $(\mathcal{S})_{-\rho}(H)$.

Proposition 3. A set $\mathcal{M}$ is bounded in $(\mathcal{S})_{-\rho}(H)$ if and only if, for any bounded subset $M$ of $(\mathcal{S})_{\rho}$, there exists a constant $K>0$ such that $\|\Phi[\varphi]\| \leq K$ for all $\varphi \in M$ and $\Phi \in \mathcal{M}$.

Proof. Necessity. Let $\mathcal{M}$ be bounded in $(\mathcal{S})_{-\rho}(H)$. Assume that there exists a set $M$ bounded in $(\mathcal{S})_{\rho}$ and such that, for any $n \in \mathbb{N}$, there exist $\varphi_{n} \in M$ and $\Phi_{n} \in \mathcal{M}$ for which $\left\|\Phi_{n}\left[\varphi_{n}\right]\right\|>n$. We have

$$
\sup _{k \in \mathbb{N}}\left\|\frac{1}{n} \Phi_{n}\left[\varphi_{k}\right]\right\| \geq\left\|\frac{1}{n} \Phi_{n}\left[\varphi_{n}\right]\right\|>1
$$

hence, $\left\{\frac{1}{n} \Phi_{n}\right\}$ does not converge to zero uniformly on the bounded set $\left\{\varphi_{k}, k \in \mathbb{N}\right\} \subseteq M$; i.e., $\left\{\frac{1}{n} \Phi_{n}\right\}$ does not converge to zero in $(\mathcal{S})_{-\rho}(H)$.

Sufficiency. The sufficiency follows from the estimate $\left\|\varepsilon_{n} \Phi[\varphi]\right\| \leq\left|\varepsilon_{n}\right| K$.

Proposition 4. If $\mathcal{M}$ is bounded in $(\mathcal{S})_{-\rho}(H)$, then, for some $p \in \mathbb{N}$ and $K>0$, the inequality $\|\Phi[\varphi]\| \leq K|\varphi|_{p, \rho}$ holds for any $\Phi \in \mathcal{M}$ and $\varphi \in(\mathcal{S})_{\rho}$.

Proof. Assume that this is not so, i.e., that, for any $p \in \mathbb{N}$, there exist $\varphi_{p} \in M$ and $\Phi_{p} \in \mathcal{M}$ for which $\left\|\Phi_{p}\left[\varphi_{p}\right]\right\|>p\left|\varphi_{p}\right|_{p, \rho}$. Consider the sequence $\left\{\frac{\varphi_{n}}{\left|\varphi_{n}\right|_{n, \rho}}\right\}$. It is bounded in $(\mathcal{S})_{\rho}$, since

$$
\left|\frac{\varphi_{n}}{\left|\varphi_{n}\right|_{n, \rho}}\right|_{p, \rho}=\frac{\left|\varphi_{n}\right|_{p, \rho}}{\left|\varphi_{n}\right|_{n, \rho}} \leq 1
$$

for any $p \in \mathbb{N}$ and $n>p$. Then, by Proposition 3 , there exists $K>0$ such that $\left\|\Phi\left[\frac{\varphi_{n}}{\left|\varphi_{n}\right|_{n, \rho}}\right]\right\| \leq K$ for all $\Phi \in \mathcal{M}$ and $n \in \mathbb{N}$, which contradicts the inequality $\left\|\Phi\left[\frac{\varphi_{n}}{\left|\varphi_{n}\right|_{n, \rho}}\right]\right\|>n$.

Thus, if a set $\mathcal{M}$ is bounded in $(\mathcal{S})_{-\rho}(H)$, then, for some $p \in \mathbb{N}$, all elements of $\mathcal{M}$ are bounded operators from $\left(\mathcal{S}_{p}\right)_{\rho}$ to $H$ and $\mathcal{M}$ is bounded in $\mathcal{L}\left(\left(\mathcal{S}_{p}\right)_{\rho}, H\right)$. This implies the following statement.

Proposition 5. If a set $\mathcal{M}$ is bounded in $(\mathcal{S})_{-\rho}(H)$, then $\mathcal{M} \subset \operatorname{HS}\left(\left(\mathcal{S}_{p}\right)_{\rho} ; H\right)$ for some $p \in \mathbb{N}$ and $\mathcal{M}$ is bounded in $\operatorname{HS}\left(\left(\mathcal{S}_{p}\right)_{\rho} ; H\right)$.

The following statement characterizes convergence in the space $(\mathcal{S})_{-\rho}(H)$.

Proposition 6. Let $\Phi_{n}=\sum_{\alpha} \Phi_{\alpha}^{(n)} \mathbf{h}_{\alpha}$, and let $\Phi=\sum_{\alpha} \Phi_{\alpha} \mathbf{h}_{\alpha} \in(\mathcal{S})_{-\rho}(H)$. The following statements are equivalent:

(i) $\left\{\Phi_{n}\right\}$ converges to $\Phi$ in the space $(\mathcal{S})_{-\rho}(H)$;

(ii) for some $p \in \mathbb{N}$, all elements of the sequence $\left\{\Phi_{n}\right\}$ and $\Phi$ belong to $\operatorname{HS}\left(\left(\mathcal{S}_{p}\right)_{\rho} ; H\right)$ and $\lim _{n \rightarrow \infty}\left\|\Phi_{n}-\Phi\right\|_{\mathrm{HS}, p, \rho}=0$.

Proof. $(i) \Rightarrow(i i)$. Let $\left\{\Phi_{n}\right\}$ converge to $\Phi$ in the space $(\mathcal{S})_{-\rho}(H)$. Then, for any $\alpha \in \mathcal{T}$, we have $\left\|\Phi_{\alpha}^{(n)}-\Phi_{\alpha}\right\|=\frac{1}{\alpha !}\left\|\Phi^{(n)}\left[\mathbf{h}_{\alpha}\right]-\Phi\left[\mathbf{h}_{\alpha}\right]\right\| \rightarrow 0$ as $n \rightarrow \infty$. By Proposition $2, \Phi \in \operatorname{HS}\left(\left(\mathcal{S}_{p}\right)_{\rho} ; H\right)$ for some $p \in \mathbb{N}$. For an arbitrary set $M$ bounded in $(\mathcal{S})_{\rho}$, the inequality $\left\|\Phi_{n}[\varphi]-\Phi[\varphi]\right\|<1$ holds starting with some $n$ for all $\varphi \in M$; hence, $\left\|\Phi_{n}[\varphi]\right\| \leq 1+\|\Phi\|_{\mathrm{HS}, p, \rho}|\varphi|_{p, \rho} \leq 1+\|\Phi\|_{\mathrm{HS}, p, \rho} K_{p}$, where $K_{p}=\sup _{\varphi \in M}|\varphi|_{p, \rho}$. Hence, by Proposition 3, the sequence $\left\{\Phi_{n}\right\}$ is bounded in $(\mathcal{S})_{-\rho}(H)$. As follows from Proposition 5, the whole sequence belongs to some space $\operatorname{HS}\left(\left(\mathcal{S}_{q}\right)_{\rho} ; H\right)$ and is bounded in it. By (2.3) and (2.4), we can assume that there exists $q$ such that, for any $p>q$, 
the sequence $\left\{\Phi_{n}\right\}$ and $\Phi$ are contained in $\operatorname{HS}\left(\left(\mathcal{S}_{p}\right)_{\rho} ; H\right)$ and elements of $\left\{\Phi_{n}\right\}$ are bounded in the norm of each of these spaces by some constant $K>0$.

Let Index $\alpha:=\max \left\{n \in \mathbb{N}, \alpha_{n} \neq 0\right\}$. The following estimate holds:

$$
\begin{gathered}
\left\|\Phi_{n}-\Phi\right\|_{\mathrm{HS}, p+1, \rho}^{2}=\sum_{\operatorname{Index} \alpha \leq k}(\alpha !)^{1-\rho}\left\|\Phi_{\alpha}^{(n)}-\Phi_{\alpha}\right\|^{2}(2 \mathbb{N})^{-2(p+1) \alpha} \\
+\sum_{\operatorname{Index} \alpha>k}(\alpha !)^{1-\rho}\left\|\Phi_{\alpha}^{(n)}-\Phi_{\alpha}\right\|^{2}(2 \mathbb{N})^{-2(p+1) \alpha} \\
\leq \max _{\operatorname{Index} \alpha \leq k}\left[(\alpha !)^{1-\rho}\left\|\Phi_{\alpha}^{(n)}-\Phi_{\alpha}\right\|^{2}\right] \times \sum_{\operatorname{Index} \alpha \leq k}(2 \mathbb{N})^{-2(p+1) \alpha} \\
\quad+\sum_{\operatorname{Index~} \alpha>k}\left[(\alpha !)^{1-\rho}\left(2\left\|\Phi_{\alpha}^{(n)}\right\|^{2}+2\left\|\Phi_{\alpha}\right\|^{2}\right)(2 \mathbb{N})^{-2 p \alpha}\right](2 \mathbb{N})^{-2 \alpha} \\
\leq \max _{\operatorname{Index} \alpha \leq k}\left[(\alpha !)^{1-\rho}\left\|\Phi_{\alpha}^{(n)}-\Phi_{\alpha}\right\|^{2}\right] \times A(2 p+1)+4 K^{2} \sum_{\operatorname{Index~} \alpha>k}(2 \mathbb{N})^{-2 \alpha} .
\end{gathered}
$$

For any $\varepsilon>0$, choose $k$ such that

$$
\sum_{\operatorname{Index} \alpha>k}(2 \mathbb{N})^{-2 \alpha}<\frac{\varepsilon}{8 K^{2}}
$$

and $N$ such that

$$
\max _{\operatorname{Index} \alpha \leq k}\left[(\alpha !)^{1-\rho}\left\|\Phi_{\alpha}^{(n)}-\Phi_{\alpha}\right\|^{2}\right]<\frac{\varepsilon}{2 A(2 p+2)}
$$

for $n>N$; then, $\left\|\Phi_{n}-\Phi\right\|_{\mathrm{HS}, p+1, \rho}^{2}<\varepsilon$ for $n>N$.

$($ ii $) \Rightarrow(i)$ This implication is obvious.

For functions $\Phi(\cdot): \mathbb{R} \rightarrow(\mathcal{S})_{-\rho}(H)$, we will understand the limit and derivative at the point $t_{0} \in \mathbb{R}$ in the sense of the uniform convergence on bounded subsets of $(\mathcal{S})_{\rho}$. The following statement is easily obtained from Proposition 6 .

Corollary 1. Let $\Phi(t)=\sum_{\alpha} \Phi_{\alpha}(t) \mathbf{h}_{\alpha} \in(\mathcal{S})_{-\rho}(H)$ for $t \in[a, b], t_{0} \in[a, b]$. Then,

(1) $\lim _{t \rightarrow t_{0}} \Phi(t)=\Phi\left(t_{0}\right)$ in the space $(\mathcal{S})_{-\rho}(H)$ if and only if, for some $p \in \mathbb{N}$, all $\Phi(t), t \in[a, b]$, belong to $\operatorname{HS}\left(\left(\mathcal{S}_{p}\right)_{\rho} ; H\right)$ and $\lim _{n \rightarrow \infty}\left\|\Phi(t)-\Phi\left(t_{0}\right)\right\|_{\mathrm{HS}, p, \rho}=0$;

(2) the function $\Phi(t)$ is differentiable at the point $t_{0} \in[a, b]$ if and only if, for some $p$, there exists $\frac{d \Phi}{d t}:=\lim _{t \rightarrow t_{0}} \frac{\Phi(t)-\Phi\left(t_{0}\right)}{t-t_{0}}$ in the space $\operatorname{HS}\left(\left(\mathcal{S}_{p}\right)_{\rho} ; H\right)$.

Example 1. (An $H$-valued cylindrical Wiener process and $H$-valued white noise.)

Let $n(\cdot, \cdot): \mathbb{N} \times \mathbb{N} \rightarrow \mathbb{N}$ be a bijection satisfying the condition

$$
n(i, j) \geq i j, \quad i, j \in \mathbb{N} .
$$

Define a sequence of independent Brownian motions $\left\{\beta_{j}(t)\right\}_{j=1}^{\infty}$ by setting

$$
\beta_{j}(t)=\sum_{i=1}^{\infty} \int_{0}^{t} \xi_{i}(s) d s \mathbf{h}_{\epsilon_{n(i, j)}},
$$


where $\epsilon_{n}:=\left(0,0, \ldots, \frac{1}{n}, 0, \ldots\right)$. Then, the $H$-valued random process defined by the equality

$$
W(t)=\sum_{j \in \mathbb{N}} \beta_{j}(t) e_{j}=\sum_{n \in \mathbb{N}} W_{\epsilon_{n}}(t) \mathbf{h}_{\epsilon_{n}}, \quad W_{\epsilon_{n}}(t)=\int_{0}^{t} \xi_{i(n)}(s) d s e_{j(n)} \in H,
$$

where $i(n), j(n) \in \mathbb{N}$ are such that $n(i(n), j(n))=n$, is called a cylindrical Wiener process.

It is easy to verify that $W(t) \notin\left(L^{2}\right)(H)$ for all $t \in \mathbb{R}$. At the same time, the known estimate $\int_{0}^{t} \xi_{i}(s) d s=O\left(i^{-\frac{3}{4}}\right)$ and condition $(2.5)$ imply that $\|W(t)\|_{\mathrm{HS}, 1, \rho}^{2}<\infty$. Thus, $W(t) \in$ $\operatorname{HS}\left(\left(\mathcal{S}_{1}\right)_{\rho} ; H\right) \subset(\mathcal{S})_{-\rho}(H)$.

Define an $H$-valued cylindrical white noise by the equality

$$
\mathbb{W}(t):=\sum_{i, j \in \mathbb{N}} \xi_{i}(t)\left(\mathbf{h}_{\epsilon_{n(i, j)}} e_{j}\right)=\sum_{n \in \mathbb{N}} \mathbb{W}_{\epsilon_{n}}(t) \mathbf{h}_{\epsilon_{n}}, \quad \mathbb{W}_{\epsilon_{n}}(t)=\xi_{i(n)}(t) e_{j(n)} \in H
$$

Since $\xi_{i}(t)=O\left(i^{-\frac{1}{4}}\right)$, we have $\|\mathbb{W}(t)\|_{\mathrm{HS}, 1, \rho}^{2}<\infty$; hence, $\mathbb{W}(t) \in \operatorname{HS}\left(\left(\mathcal{S}_{1}\right)_{\rho} ; H\right) \subset(\mathcal{S})_{-\rho}(H)$.

Note that $\frac{d}{d t} W(t)=\mathbb{W}(t)$ for all $t \in \mathbb{R}$.

2.3. An $S$-transformation of $H$-valued generalized random variables. Let $\mathcal{E}_{h}:=$ $e^{\langle\cdot, h\rangle-\frac{1}{2}|h|_{0}^{2}}$. For $h \in \mathcal{S}$, this random variable is defined on the whole space $\mathcal{S}^{\prime}$ and belongs to $(\mathcal{S})_{\rho}$ for $0 \leq \rho<1$; moreover,

$$
\left|\mathcal{E}_{h}\right|_{p, \rho} \leq 2^{\rho / 2} \exp \left[(1-\rho)^{\frac{2 \rho-1}{1-\rho}}|h|_{p}^{\frac{2}{1-\rho}}\right]
$$

(see, for example, [2]). The expansion of $\mathcal{E}_{h}$ into a series in stochastic Hermite polynomials has the form

$$
\mathcal{E}_{h}=\sum_{\alpha \in \mathcal{T}} e_{\alpha} \mathbf{h}_{\alpha}, \quad e_{\alpha}=\frac{1}{\alpha !} \prod_{i=1}^{\infty}\left(h, \xi_{i}\right)_{L^{2}(\mathbb{R})}^{\alpha_{i}} .
$$

Indeed, let $h \in \mathcal{S}$, and let $\mathbf{h}_{\alpha}=\prod_{i=1}^{n} h_{\alpha_{i}}\left(\left\langle\cdot, \xi_{i}\right\rangle\right)$ be a stochastic Hermite polynomial. Using the expansion $h=\sum_{i=1}^{n}\left(h, \xi_{i}\right)_{L^{2}(\mathbb{R})} \xi_{i}+h^{\perp}$, relation $(2.2)$, and the properties of the generating function of Hermite polynomials, we obtain

$$
\begin{gathered}
e_{\alpha}=\left(\mathcal{E}_{h}, \mathbf{h}_{\alpha}\right)_{\left(L^{2}\right)}=\int_{\mathcal{S}^{\prime}} e^{\langle\omega, h\rangle-\frac{1}{2}|h|_{0}^{2}} \mathbf{h}_{\alpha}(\omega) d \mu(\omega) \\
=\int_{\mathcal{S}^{\prime}} e^{\sum_{i=1}^{n}\left\langle\omega, \xi_{i}\right\rangle\left(h, \xi_{i}\right)_{L^{2}(\mathbb{R})}+\left\langle\omega, \frac{h^{\perp}}{\left|h^{\perp}\right|_{0}}\right\rangle\left|h^{\perp}\right|_{0}-\frac{1}{2}\left(\sum_{i=1}^{n}\left(h, \xi_{i}\right)_{L^{2}(\mathbb{R})}+\left|h^{\perp}\right|_{0}^{2}\right)} \prod_{i=1}^{n} h_{\alpha_{i}}\left(\left\langle\omega, \xi_{i}\right\rangle\right) d \mu(\omega) \\
=\frac{1}{(2 \pi)^{\frac{n+1}{2}}} \int_{\mathbb{R}^{n+1}} e^{\sum_{i=1}^{n} x_{i}\left(h, \xi_{i}\right)+x_{n+1}\left|h^{\perp}\right|_{0}-\frac{1}{2}\left(\sum_{i=1}^{n}\left(h, \xi_{i}\right)^{2}+\left|h^{\perp}\right|_{0}^{2}\right)} \prod_{i=1}^{n} h_{\alpha_{i}}\left(x_{i}\right) e^{-\frac{1}{2} \sum_{i=1}^{n+1} x_{i}^{2}} d x_{1} \ldots d x_{n+1} \\
=\prod_{i=1}^{n} \frac{1}{\sqrt{2 \pi}} \int_{\mathbb{R}} e^{x_{i}\left(h, \xi_{i}\right)-\frac{1}{2}\left(h, \xi_{i}\right)^{2}} h_{\alpha_{i}}\left(x_{i}\right) e^{-\frac{1}{2} x_{i}^{2}} d x_{i} \frac{1}{\sqrt{2 \pi}} \int_{\mathbb{R}} e^{x\left|h^{\perp}\right|_{0}-\frac{1}{2}\left|h^{\perp}\right|_{0}^{2}-\frac{1}{2} x^{2}} d x=\prod_{i=1}^{n} \frac{\left(h, \xi_{i}\right)_{L^{2}(\mathbb{R})}^{\alpha_{i}}}{\alpha_{i} !} .
\end{gathered}
$$

PROCEEDINGS OF THE STEKLOV INSTITUTE OF MATHEMATICS $\quad$ Vol. $280 \quad$ Suppl. $1 \quad 2013$ 
Let $\Phi \in(\mathcal{S})_{-\rho}(H), 0 \leq \rho<1$. The $S$-transform of $\Phi$ is the $H$-valued function

$$
(S \Phi)(h)=\Phi\left[\mathcal{E}_{h}\right], \quad h \in \mathcal{S} .
$$

It follows from estimate $(2.6)$ that, for any $\Phi \in(\mathcal{S})_{-\rho}(H)$, there exists $p \in \mathbb{N}$ such that

$$
\|(S \Phi)(h)\|=\left\|\Phi\left[\mathcal{E}_{h}\right]\right\| \leq 2^{\rho / 2}\|\Phi\|_{H S, p, \rho} \exp \left[(1-\rho)^{\frac{2 \rho-1}{1-\rho}}|h|_{p}^{\frac{2}{1-\rho}}\right] .
$$

It turns out that an estimate of type (2.8) is sufficient for a function from $\mathcal{S}$ to $H$ to be an $S$-transform of some generalized $H$-valued random variable. More exactly, the following characteristic theorem is valid; its proof repeats the proof of the similar theorem for the $\mathbb{R}$-valued case almost word-for-word (see, for example, [2]).

Theorem 1. Let $\Phi \in(\mathcal{S})_{-\rho}(H), 0 \leq \rho<1$. Then, the function $F=S \Phi$ satisfies the following conditions:

(i) For any $h, \theta \in \mathcal{S}$, the function $F(h+z \theta)$ is an entire analytic function of the variable $z \in \mathbb{C}$.

(ii) There exist $K>0, a>0$, and $p \in \mathbb{N}$ such that

$$
\|F(h)\| \leq K \exp \left[a|h|_{p}^{\frac{2}{1-\rho}}\right], \quad h \in \mathcal{S} .
$$

If a function $F: \mathcal{S} \rightarrow H$ satisfies conditions $(i)$ and $(i i)$, then there exists a unique function $\Phi \in(\mathcal{S})_{-\rho}(H)$ for which $F=S \Phi$. In this case, for any $q$ such that $e^{2}\left(\frac{2 a}{1-\rho}\right)^{1-\rho} \sum_{i=1}^{\infty}(2 i)^{-2(q-p)}<1$, the following estimate holds:

$$
\|\Phi\|_{\mathrm{HS}, q, \rho} \leq K\left(1-e^{2}\left(\frac{2 a}{1-\rho}\right)^{1-\rho} \sum_{i=1}^{\infty}(2 i)^{-2(q-p)}\right)^{-1 / 2} .
$$

Example 2. Let $(S \mathbb{W}(t))(h)=\mathbb{W}(t)\left[\mathcal{E}_{h}\right]=\sum_{i, j \in \mathbb{N}} \xi_{i}(t) e_{j}\left(\xi_{n(i, j)}, h\right)_{L^{2}(\mathbb{R})}$. Since the functions $\xi_{i}(t) e_{j}, i, j \in \mathbb{N}$, form an orthonormal basis of the space $L^{2}(\mathbb{R} ; H)$, we have

$$
\|[S \mathbb{W}(\cdot)](h)\|_{L^{2}(\mathbb{R} ; H)}^{2}=\sum_{i, j \in \mathbb{N}}\left|\left(\xi_{n(i, j)}, h\right)_{L^{2}(\mathbb{R})}\right|^{2}=|h|_{L^{2}(\mathbb{R})}^{2} .
$$

2.4. The Wick product. Let $H_{1}$ and $H_{2}$ be separable Hilbert spaces. Then, $\operatorname{HS}\left(H_{1} ; H_{2}\right)$, i.e., the space of Hilbert-Schmidt operators from $H_{1}$ to $H_{2}$, is a separable Hilbert space. Hence, we can consider the space $(\mathcal{S})_{-\rho}\left(\mathrm{HS}\left(H_{1} ; H_{2}\right)\right)$ of generalized random variables with values in $\operatorname{HS}\left(H_{1} ; H_{2}\right)$ over $(\mathcal{S})_{\rho}$, as this is done in Subsection 2.2. Let $\Psi \in(\mathcal{S})_{-\rho}\left(\operatorname{HS}\left(H_{1} ; H_{2}\right)\right)$, and let $\Phi \in(\mathcal{S})_{-\rho}\left(H_{1}\right)$. Their $S$-transforms satisfy conditions $(i)$ and $(i i)$ of Theorem 1 . For any $h \in \mathcal{S}$, we have $S \Psi(h) \in \operatorname{HS}\left(H_{1} ; H_{2}\right)$ and $S \Phi(h) \in H_{1}$; hence, $F(h)=S \Psi(h) S \Phi(h) \in H_{2}$, and $F(h+z \theta)$ is an entire analytic function of the variable $z \in \mathbb{C}$ for any $h, \theta \in \mathcal{S}$. Moreover,

$$
\|S \Psi(h) S \Phi(h)\|_{H_{2}} \leq\|S \Psi(h)\|_{\mathrm{HS}\left(H_{1} ; H_{2}\right)}\|S \Phi(h)\|_{H_{1}} \leq K_{1} K_{2} \exp \left[\left(a_{1}+a_{2}\right)|h|_{p}^{\frac{2}{1-\rho}}\right],
$$

where $K_{1}, K_{2}, a_{1}, a_{2}$ are the constants from condition $(i i)$ of Theorem 1 for the functions $\Psi$ and $\Phi$, respectively (evidently, we can assume that these conditions are satisfied with the same constant $p$ ).

PROCEEDINGS OF THE STEKLOV INSTITUTE OF MATHEMATICS ～Vol. $280 \quad$ Suppl. $1 \quad 2013$ 
Consequently, $F$ is the $S$-transform of some generalized random variable $\Theta \in(\mathcal{S})_{-\rho}\left(H_{2}\right)$. This validates the following definition.

Let $\Psi \in(\mathcal{S})_{-\rho}\left(\operatorname{HS}\left(H_{1} ; H_{2}\right)\right)$ and $\Phi \in(\mathcal{S})_{-\rho}\left(H_{1}\right)$. The generalized random variable $\Theta \in$ $(\mathcal{S})_{-\rho}\left(H_{2}\right)$ such that $S \Theta=S \Psi S \Phi$ is called the Wick product of $\Psi$ and $\Phi$ and is denoted by $\Psi \diamond \Phi$.

Expansion (2.7) implies the equalities

$$
S \Psi(h)=\sum_{\alpha \in \mathcal{T}} \Psi_{\alpha} \prod_{i=1}^{\infty}\left(h, \xi_{i}\right)_{L^{2}(\mathbb{R})}^{\alpha_{i}}, \quad S \Phi(h)=\sum_{\alpha \in \mathcal{T}} \Phi_{\alpha} \prod_{i=1}^{\infty}\left(h, \xi_{i}\right)_{L^{2}(\mathbb{R})}^{\alpha_{i}}
$$

Multiplying these expansions, we obtain

$$
S \Psi(h) S \Phi(h)=\sum_{\gamma \in \mathcal{T}}\left(\sum_{\alpha+\beta=\gamma} \Psi_{\alpha} \Phi_{\beta}\right) \prod_{i=1}^{\infty}\left(h, \xi_{i}\right)_{L^{2}(\mathbb{R})}^{\gamma_{i}} .
$$

By the uniqueness of the $S$-transform, we get

$$
\Psi \diamond \Phi=\sum_{\gamma \in \mathcal{T}}\left(\sum_{\alpha+\beta=\gamma} \Psi_{\alpha} \Phi_{\beta}\right) \mathbf{h}_{\alpha}
$$

2.5. The Hitsuda-Skorokhod integral. Let $Q \in \operatorname{HS}(H ; H)$. Denote by $H_{Q}$ the space $Q^{\frac{1}{2}}(H)$ with scalar product $(u, v)_{H_{Q}}=\left(Q^{-\frac{1}{2}} u, Q^{-\frac{1}{2}} v\right)_{H}$. The following statement holds for the $H$-valued cylindrical white noise defined above.

Proposition 7. For any $t \in \mathbb{R}$ and $\rho \in[0 ; 1]$, the inclusion $\mathbb{W}(t) \in(\mathcal{S})_{-\rho}\left(H_{Q}\right)$ holds for any operator $Q=\sum_{j=1}^{\infty} \sigma_{j}^{2}\left(e_{j} \otimes e_{j}\right) \in \operatorname{HS}(H ; H),{ }^{3}$ i.e., for any operator such that $\sum_{j=1}^{\infty} \sigma_{j}^{2}<\infty$, if

$$
\sum_{j=1}^{\infty} \sigma_{j}^{-2} j^{-2 p}<\infty \text { for some } p \in \mathbb{N}
$$

Proof. The validity of the proposition follows from the estimate

$$
\left\|\mathbb{W}_{\epsilon_{n(i, j)}}\right\|_{H_{Q}}^{2}(2 \mathbb{N})^{-2 p \epsilon_{n(i, j)}}=\left|\xi_{i}(t)\right|^{2} \sigma_{j}^{-2}(2 n(i, j))^{-2 p} \leq \frac{\left|\xi_{i}(t)\right|^{2}}{\sigma_{j}^{2}(2 i j)^{2 p}}=O\left(\sigma_{j}^{-2} i^{-2 p-\frac{1}{2}} j^{-2 p}\right) .
$$

As follows from Proposition 7, if $Q$ satisfies condition $(2.9)$, then the $(\mathcal{S})_{-1}(H)$-valued random process $\Psi(t) \diamond \mathbb{W}(t)$ is defined for any $(\mathcal{S})_{-\rho}\left(\operatorname{HS}\left(H_{Q} ; H\right)\right)$-valued random process $\Psi(t)$.

$\operatorname{An}(\mathcal{S})_{-\rho}\left(\operatorname{HS}\left(H_{Q} ; H\right)\right)$-valued random process $\Psi(t)$ is called Hitsuda-Skorokhod integrable on the interval $[0 ; T]$ if the process $\Psi(t) \diamond \mathbb{W}(t)$ is integrable on $[0 ; T]$ as an $(\mathcal{S})_{-1}(H)$-valued function. In this case, the integral

$$
\int_{0}^{T} \Psi(t) \diamond \mathbb{W}(t) d t
$$

is called the Hitsuda-Skorokhod integral of $\Psi(t)$.

\footnotetext{
${ }^{3}$ For $v \in V$ and $u \in U$, where $V$ and $U$ are Hilbert spaces, we denote by $v \otimes u$ the operator from $U$ in $V$ defined by the equality $(v \otimes u) h:=v(u, h)_{U}$.
}

PROCEEDINGS OF THE STEKLOV INSTITUTE OF MATHEMATICS ～Vol. 280 Suppl. 12013 
The Hitsuda-Skorokhod integral is a generalization of the Ito integral $\int_{0}^{T} \Psi(t) d W(t)$ with respect to the cylindrical Wiener process in the sense that, if

$$
\Psi(t) \in\left(L^{2}\right)\left(\operatorname{HS}\left(H_{Q} ; H\right)\right)
$$

for all $t \in[0 ; T]$ and $\Psi(t)$ is adapted to the filtration generated by the process $W(t)$, then

$$
\int_{0}^{T} \Psi(t) \diamond \mathbb{W}(t) d t=\int_{0}^{T} \Psi(t) d W(t) .
$$

\section{THE CAUCHY PROBLEM FOR A LINEAR DIFFERENTIAL OPERATOR EQUATION WITH MULTIPLICATIVE NOISE}

Let $A$ be a linear operator from a Hilbert space $H_{1}$ to a Hilbert space $H_{2}$. We define its action as an operator from $(\mathcal{S})_{-\rho}\left(H_{1}\right)$ to $(\mathcal{S})_{-\rho}\left(H_{2}\right)$ as follows. If $A \in \mathcal{L}\left(H_{1}, H_{2}\right)$, let

$$
A \Phi:=\sum_{\alpha \in \mathcal{T}} A \Phi_{\alpha} \mathbf{h}_{\alpha} \text { for } \Phi=\sum_{\alpha \in \mathcal{T}} \Phi_{\alpha} \mathbf{h}_{\alpha} \in(\mathcal{S})_{-\rho}\left(H_{1}\right) .
$$

The operator $A$ defined in this way, evidently, is a linear continuous operator from $(\mathcal{S})_{-\rho}\left(H_{1}\right)$ to $(\mathcal{S})_{-\rho}\left(H_{2}\right)$. If $A$ is unbounded, we define $(\operatorname{dom} A)$ as the set of all

$$
\sum_{\alpha \in \mathcal{T}} \Phi_{\alpha} \mathbf{h}_{\alpha} \in(\mathcal{S})_{-\rho}\left(H_{1}\right)
$$

for which $\Phi_{\alpha} \in \operatorname{dom} A$ for all $\alpha \in \mathcal{T}$ and

$$
\sum_{\alpha \in \mathcal{T}}(\alpha !)^{1-\rho}\left\|A \Phi_{\alpha}\right\|_{H_{2}}^{2}(2 \mathbb{N})^{-2 p \alpha}<\infty \text { for some } p \in \mathbb{N}
$$

Then, formula $(3.1)$ defines on $(\operatorname{dom} A)$ a linear operator from $(\mathcal{S})_{-\rho}\left(H_{1}\right)$ to $(\mathcal{S})_{-\rho}\left(H_{2}\right)$. It is easy to verify that this operator is closed if $A$ is a closed operator from $H_{1}$ to $H_{2}$.

Proposition 8. Let $A$ be a closed operator from $H_{1}$ to $H_{2}$. For any $\Phi \in(\operatorname{dom} A) \subseteq(\mathcal{S})_{-\rho}\left(H_{1}\right)$, $[S \Phi](h) \in \operatorname{dom} A \subseteq H_{1}$ and

$$
[S A \Phi](h)=A[S \Phi](h), \quad h \in \mathcal{S} .
$$

Assume that $A$ is a linear closed operator acting in the space $H, B(\cdot) \in \mathcal{L}(H, \mathcal{L}(H))$, and $\Phi \in(\operatorname{dom} A) \subseteq(\mathcal{S})_{-\rho}(H)$. Consider Cauchy problem $(1.2)$

$$
\frac{d X(t)}{d t}=A X(t)+B(X(t)) \diamond \mathbb{W}(t), \quad t \geq 0, \quad X(0)=\Phi .
$$

It is obtained by replacing the Ito integral in equation (1.1) by the Hitsuda-Skorokhod integral and differentiating both sides with respect to $t$. Consider the question on the existence of a solution to problem $(1.2)$ in the space $(\mathcal{S})_{-\rho}(H)$ for $\rho \in[0 ; 1)$, i.e., the existence of an $(\mathcal{S})_{-\rho}(H)$-valued differentiable function satisfying (1.2). Note that, if $Q$ is a kernel operator in $H$ and satisfies the assumption of Proposition 7 for some $p \in \mathbb{N}$, then the Wick product in (1.2) is defined because $B(X(t)) \in(\mathcal{S})_{-\rho}\left(\operatorname{HS}\left(H_{Q} ; H\right)\right)$ for any $X(t) \in(\mathcal{S})_{-\rho}(H)$. 
Applying the $S$-transformation to problem (1.2), we obtain the following problem:

$$
\frac{d}{d t} \hat{X}(t, h)=A \hat{X}(t, h)+B(\hat{X}(t, h)) \hat{\mathbb{W}}(t, h), \quad t \geq 0, \quad \hat{X}(0, h)=\hat{\Phi}(h), \quad h \in \mathcal{S},
$$

where $\hat{X}(t, h)=S[X(t)](h), \hat{\mathbb{W}}(t, h)=S[\mathbb{W}(t)](h)$, and $\hat{\Phi}(h)=S \Phi(h)$.

In what follows, we assume that the operator $B$ satisfies the following condition.

Conjecture 1. For any $y \in H$,

(BI) $B(\operatorname{dom} A) y \subseteq \operatorname{dom} A$;

(BII) the operator $C(\cdot) y: \operatorname{dom} A \rightarrow \mathcal{L}(H)$ defined by the equality

$$
C(x) y:=A B(x) y-B(A x) y, \quad x \in \operatorname{dom} A,
$$

is bounded.

By the uniform boundedness principle, Conjecture 1 implies that there is $M_{A B}>0$ such that

$$
\|C(x) y\| \leq M_{A B}\|x\|\|y\|, \quad x \in \operatorname{dom} A, \quad y \in H .
$$

Let the operator $A$ generate a semigroup $\{U(t), t \geq 0\}$ of class $C_{0}$. We will assume that constants $M>0$ and $a \in \mathbb{R}$ are such that

$$
\|U(t)\| \leq M e^{a t}, \quad t \geq 0 .
$$

Proposition 9. If $\operatorname{ker} B(\cdot) y=\{0\}$ for all $y \in H$, then problem (3.2) has at most one solution.

Proof. If a function $\hat{X}(\cdot, h)$ solves problem $(3.2)$ for some $h \in \mathcal{S}$, then it solves the equation

$$
\hat{X}(t, h)=U(t) \hat{\Phi}(h)+\int_{0}^{t} U(t-s) B(\hat{X}(s, h)) \hat{\mathbb{W}}(s, h) d s, \quad t \geq 0 .
$$

(The converse, in general, is not true.) Thus, to prove the uniqueness of solution to problem (3.2), it is sufficient to prove that the equation

$$
\int_{0}^{t} U(t-s) B(\hat{X}(s, h)) \hat{\mathbb{W}}(s, h) d s=0, \quad t \geq 0,
$$

has only trivial solution $X(\cdot, h) \equiv 0$ on $[0 ; \infty)$ for any $h \in H$. For this, we apply the Laplace transformation to both sides of this equation. For $\operatorname{Re} \lambda>a$, we have

$$
\begin{aligned}
& \int_{0}^{\infty} d t e^{\lambda t} \int_{0}^{t} U(t-s) B(\hat{X}(s, h)) \hat{\mathbb{W}}(s, h) d s=\int_{0}^{\infty} d s \int_{s}^{\infty} e^{\lambda t} U(t-s) B(\hat{X}(s, h)) \hat{\mathbb{W}}(s, h) d t \\
& =\int_{0}^{\infty} d s \int_{0}^{\infty} e^{\lambda(t+s)} U(t) B(\hat{X}(s, h)) \hat{\mathbb{W}}(s, h) d t=(\lambda-A)^{-1} \int_{0}^{\infty} e^{\lambda s} B(\hat{X}(s, h)) \hat{\mathbb{W}}(s, h) d s .
\end{aligned}
$$

By the properties of the resolvent of $A$ and of the Laplace transform, we conclude that, if $\hat{X}(\cdot, h)$ solves equation $(3.5)$ for any $h \in \mathcal{S}$, then $B(\hat{X}(\cdot, h)) \hat{\mathbb{W}}(\cdot, h) \equiv 0$ on $[0 ; \infty)$ for any $h \in \mathcal{S}$. Setting 
$h=\xi_{n(i, j)}, i, j \in \mathbb{N}$, we obtain $B(\hat{X}(\cdot, h)) \hat{\mathbb{W}}(\cdot, h)=B\left(\xi_{i}(\cdot) \hat{X}\left(\cdot, \xi_{n(i, j)}\right)\right) e_{j} \equiv 0$ on $[0 ; \infty)$, which implies that $\hat{X}(\cdot, h) \equiv 0$ on $[0 ; \infty)$ for all $h \in \mathcal{S}$.

We define a sequence of operators $\left\{T_{k}(t, h)\right\}, t \geq 0, h \in \mathcal{S}$ as follows:

$$
\begin{aligned}
T_{0}(t, h) & =U(t) \\
T_{k}(t, h) x & =\int_{0}^{t} U(t-s) B\left(T_{k-1}(s, h) x\right) \hat{\mathbb{W}}(s, h) d s, \quad x \in H, \quad k=1,2, \ldots .
\end{aligned}
$$

Lemma 1. For any $t \geq 0, h \in \mathcal{S}$, and $k \in \mathbb{N} \cup\{0\}$,

$$
\left\|T_{k}(t, h)\right\|_{\mathcal{L}(H)} \leq M^{k+1}\|B\|^{k} e^{a t}|h|_{L^{2}(\mathbb{R})}^{k} \sqrt{\frac{t^{k}}{k !}}
$$

where $M>0$ and $a \in \mathbb{R}$ are the constants from estimate (3.4) and $\|B\|=\|B\|_{\mathcal{L}(H, \mathcal{L}(H)) \text {. }}$

Proof. Assume that (3.6) holds for some $k \in \mathbb{N}$. Then, for any $x \in H$, we have

$$
\begin{gathered}
\left\|T_{k+1}(t, h) x\right\|=\left\|\int_{0}^{t} U(t-s) B\left(T_{k}(s, h) x\right) \hat{\mathbb{W}}(s, h) d s\right\| \leq \int_{0}^{t}\left\|U(t-s) B\left(T_{k}(s, h) x\right) \hat{\mathbb{W}}(s, h)\right\| d s \\
\leq M\|B\| \int_{0}^{t} e^{a(t-s)}\left\|T_{k}(s, z) x\right\|\|\hat{\mathbb{W}}(s, h)\| d s \leq M^{k+2}\|B\|^{k+1} e^{a t}|h|_{L^{2}(\mathbb{R})}^{k} \int_{0}^{t} \sqrt{\frac{s^{k}}{k !}}\|\hat{\mathbb{W}}(s, h)\| d s\|x\| \\
\leq M^{k+2}\|B\|^{k+1} e^{a t}|h|_{L^{2}(\mathbb{R})}^{k}\left(\int_{0}^{t} \frac{s^{k}}{k !} d s\right)^{1 / 2}\left(\int_{0}^{t}\|\hat{\mathbb{W}}(s, h)\|^{2} d s\right)^{1 / 2}\|x\| \\
\leq M^{k+2}\|B\|^{k+1} e^{a t}|h|_{L^{2}(\mathbb{R})}^{k} \sqrt{\frac{t^{k+1}}{(k+1) !}}\|\hat{\mathbb{W}}(\cdot, h)\|_{L^{2}(\mathbb{R} ; H)}\|x\| \leq M^{k+2}\|B\|^{k+1} e^{a t}|h|_{L^{2}(\mathbb{R})}^{k+1} \sqrt{\frac{t^{k+1}}{(k+1) !}\|x\| .}
\end{gathered}
$$

Since estimate (3.6) is valid for $k=0$, we conclude by induction that it is valid for all $k \in \mathbb{N}$.

Lemma 2. For any $t \geq 0, h \in \mathcal{S}, k \in \mathbb{N} \cup\{0\}$, and $\Phi \in(\operatorname{dom} A)$, the estimate

$$
\left\|A T_{k}(t, h) \hat{\Phi}(h)\right\| \leq M^{k+1}\|B\|^{k-1}|h|_{L^{2}(\mathbb{R})}^{k} e^{a t} \sqrt{\frac{t^{k}}{k !}}\left(\|B\|\|A \hat{\Phi}(h)\|+k M_{A B}\|\hat{\Phi}(h)\|\right)
$$

is valid, where $M>0$ and $a \in \mathbb{R}$ are the constants from estimate $(3.4),\|B\|=\|B\|_{\mathcal{L}(H, \mathcal{L}(H))}$, and $M_{A B}$ is the constant from estimate (3.3).

Proof. For $k=0$, using the properties of semigroups of class $C_{0}$, we obtain

$$
\left\|A T_{0}(t, h) \hat{\Phi}(h)\right\|=\|A U(t) \hat{\Phi}(h)\|=\|U(t) A \hat{\Phi}(h)\| \leq M e^{a t}\|\hat{\Phi}(h)\| .
$$

Further,

$$
A T_{k}(t, h) \hat{\Phi}(h)=\int_{0}^{t} A U(t-s) B\left(T_{k-1}(s, h) \hat{\Phi}(h)\right) \hat{\mathbb{W}}(s, h) d s
$$




$$
\begin{gathered}
=\int_{0}^{t} U(t-s) A B\left(T_{k-1}(s, h) \hat{\Phi}(h)\right) \hat{\mathbb{W}}(s, h) d s \\
=\int_{0}^{t} U(t-s)\left[B\left(A T_{k-1}(s, h) \hat{\Phi}(h)\right) \hat{\mathbb{W}}(s, h)+C\left(T_{k-1}(s, h) \hat{\Phi}(h)\right) \hat{\mathbb{W}}(s, h)\right] d s .
\end{gathered}
$$

If (3.7) holds for some $k \in \mathbb{N}$, then, using this representation and estimate (3.6), we obtain

$$
\begin{gathered}
\left\|A T_{k+1}(t, h) \hat{\Phi}(h)\right\| \\
\leq \int_{0}^{t} M e^{a(t-s)}\left[M^{k+1}\|B\|^{k}|h|_{L_{2}(\mathbb{R})}^{k} e^{a s} \sqrt{\frac{s^{k}}{k !}}\left(\|B\|\|A \hat{\Phi}(h)\|+k M_{A B}\|\hat{\Phi}(h)\|\right)\|\hat{\mathbb{W}}(s, h)\|\right. \\
+M_{A B} M^{k+1}\|B\|^{k} e^{a s}|h|_{L_{2}(\mathbb{R})}^{k} \sqrt{\left.\frac{s^{k}}{k !}\|\hat{\Phi}(h)\|\|\hat{\mathbb{V}}(s, h)\|\right] d s} \\
=M^{k+2}\|B\|^{k}|h|_{L_{2}(\mathbb{R})}^{k} e^{a t}\left(\|B\|\|A \hat{\Phi}(h)\|+(k+1) M_{A B}\|\hat{\Phi}(h)\|\right) \int_{0}^{t} \sqrt{\frac{s^{k}}{k !}\|\hat{\mathbb{W}}(s, h)\| d s} \\
\leq M^{k+2}\|B\|^{k}|h|_{L_{2}(\mathbb{R})}^{k} e^{a t}\left(\|B\|\|A \hat{\Phi}(h)\|+(k+1) M_{A B}\|\hat{\Phi}(h)\|\right)\left(\int_{0}^{t} \frac{s^{k}}{k !} d s\right)^{1 / 2}\left(\int_{0}^{t}\|\hat{\mathbb{W}}(s, h)\|^{2} d s\right)^{1 / 2} \\
\leq M^{k+2}\|B\|^{k}|h|_{L^{2}(\mathbb{R})}^{k+1} e^{a t} \sqrt{\frac{t^{k+1}}{(k+1) !}}\left(\|B\|\|A \hat{\Phi}(h)\|+(k+1) M_{A B}\|\hat{\Phi}(h)\|\right) .
\end{gathered}
$$

By induction, from this and (3.8), we obtain the statement of the lemma.

Consider the series

$$
T(t, h)=\sum_{k=0}^{\infty} T_{k}(t, h) .
$$

As follows from Lemma 1, the estimate

$$
\begin{aligned}
& \sum_{k=n}^{n+m}\left\|T_{k}(t, h)\right\| \leq M e^{a t} \sum_{k=n}^{n+m} \frac{\left(M \sqrt{2}\|B\||h|_{L^{2}(\mathbb{R})} \sqrt{t}\right)^{k}}{\sqrt{k !}} \times \frac{1}{\sqrt{2^{k}}} \\
& \leq M e^{a t}\left(\sum_{k=n}^{n+m} \frac{\left(2 M^{2}\|B\|^{2}|h|_{L^{2}(\mathbb{R})}^{2} t\right)^{k}}{k !}\right)^{1 / 2}\left(\sum_{k=n}^{n+m} \frac{1}{2^{k}}\right)^{1 / 2}
\end{aligned}
$$

holds for any $n, m \in \mathbb{N}$, which implies the absolute convergence of series $(3.9)$ in $\mathcal{L}(H)$ for any $t \geq 0$ and $h \in \mathcal{S}$. Thus, $T(t, h) \in \mathcal{L}(H)$.

Proposition 10. For any $\Phi \in(\operatorname{dom} A)$ and $h \in \mathcal{S}$, the function $\hat{X}(t, h)=T(t, h) \hat{\Phi}(h)$ solves problem (3.2).

Proof. For any $\Phi \in(\operatorname{dom} A)$, using Proposition 8 and the properties of $C_{0}$-semigroups, we obtain $T_{0}(t, h) \hat{\Phi}(h) \in \operatorname{dom} A$ for all $t \geq 0$ and $h \in \mathcal{S}$. As follows from condition (BI), 
$B(\operatorname{dom} A) \hat{W}(t, h) \subseteq \operatorname{dom} A$ for all $t \geq 0$ and $h \in \mathcal{S}$. Hence, we conclude by induction that $T_{k}(t, h) \hat{\Phi}(h) \in \operatorname{dom} A$ for any $\Phi \in(\operatorname{dom} A), k \in \mathbb{N}, t \geq 0$, and $h \in \mathcal{S}$. Condition (BI) also implies that $B\left(T_{k}(s, h) \hat{\Phi}(h)\right) \hat{\mathbb{W}}(t, h) \in \operatorname{dom} A$. In addition,

$$
\frac{d}{d t} U(t-s) B\left(T_{k}(s, h) \hat{\Phi}(h)\right) \hat{\mathbb{W}}(t, h)=A U(t-s) B\left(T_{k}(s, h) \hat{\Phi}(h)\right) \hat{\mathbb{W}}(t, h), \quad t \geq 0, \quad h \in \mathcal{S} .
$$

Thus, for $\Phi \in(\operatorname{dom} A)$,

$$
\begin{gathered}
\frac{d}{d t} T_{0}(t, h) \hat{\Phi}(h)=A T_{0}(t, h) \hat{\Phi}(h), \\
\frac{d}{d t} T_{k}(t, h) \hat{\Phi}(h)=\int_{0}^{t} A U(t-s) B\left(T_{k-1}(s, h) \hat{\Phi}(h)\right) \hat{\mathbb{W}}(s, h) d s+B\left(T_{k-1}(t, h) \hat{\Phi}(h)\right) \hat{\mathbb{W}}(t, h) .
\end{gathered}
$$

Since the operator $A$ is closed, we can rewrite equality (3.12) in the form

$$
\frac{d}{d t} T_{k}(t, h) \hat{\Phi}(h)=A T_{k}(t, h) \hat{\Phi}(h)+B\left(T_{k-1}(t, h) \hat{\Phi}(h)\right) \hat{\mathbb{W}}(t, h) .
$$

Using Lemma 2, we obtain the estimate

$$
\begin{gathered}
\sum_{k=n+1}^{m}\left\|A T_{k}(t, h) \hat{\Phi}(h)\right\| \leq M e^{a t}\left(\sum_{k=n+1}^{m} \frac{\left(\sqrt{2} M\|B\||h|_{L^{2}(\mathbb{R})} \sqrt{t}\right)^{k}}{\sqrt{k !}} \times \frac{1}{\sqrt{2^{k}}}\right)\|A \hat{\Phi}(h)\| \\
+\frac{M}{\|B\|} e^{a t}\left(\sum_{k=n+1}^{m} \frac{\left(\sqrt{2} M\|B\||h|_{L^{2}(\mathbb{R})} \sqrt{t}\right)^{k}}{\sqrt{k !}} \times \frac{k}{\sqrt{2^{k}}}\right) M_{A B}\|\hat{\Phi}(h)\| \\
\leq M e^{a t}\left(\sum_{k=n+1}^{m} \frac{\left(2 M^{2}\|B\|^{2}|h|_{L^{2}(\mathbb{R})^{t}}^{2}\right)^{k}}{k !}\left(\sum_{k=n+1}^{m} \frac{1}{2^{k}}\right)^{1 / 2}\|A \hat{\Phi}(h)\|\right. \\
+\frac{M}{\|B\|} e^{a t}\left(\sum_{k=n+1}^{m} \frac{\left(2 M^{2}\|B\|^{2}|h|_{L^{2}(\mathbb{R})}^{2} t\right)^{k}}{k !}\right)^{1 / 2}\left(\sum_{k=n+1}^{m} \frac{k^{2}}{2^{k}}\right)^{1 / 2} M_{A B}\|\hat{\Phi}(h)\|,
\end{gathered}
$$

which implies the convergence of the series $\sum_{k=0}^{\infty} A T_{k}(t, h) \hat{\Phi}(h)$ in the space $H$ for all $h \in \mathcal{S}$ and $\Phi \in(\operatorname{dom} A)$. As a result, summing equalities (3.11) and (3.13) over all $k \in \mathbb{N}$, we obtain on the right-hand side a series convergent in $H$ for all $t \geq 0$ and $h \in \mathcal{S}$; hence, we have proved that $\hat{X}(t, h)=T(t, h) \hat{\Phi}(h)$ is a solution of problem (3.2).

Theorem 2. Suppose that a linear closed operator $A$ whose domain is dense in $H$ generates a semigroup of class $C_{0}$, a linear bounded operator $B(\cdot): H \rightarrow \mathcal{L}(H)$ satisfies Conjecture 1 , and $\operatorname{ker} B(\cdot) y=\{0\}$ for all $y \in H$. Then, for any $\Phi \in(\operatorname{dom} A) \subseteq(\mathcal{S})_{-0}(H)$, Cauchy problem (1.2) has a unique solution in the space $(\mathcal{S})_{-0}(H)$.

Proof. As follows from Propositions 9 and 10, under the assumption of the theorem, transformed problem (3.2) has a unique solution $\hat{X}(t, h)=T(t, h) \hat{\Phi}(h)$ for any $\Phi \in(\operatorname{dom} A)$ and $h \in \mathcal{S}$. Moreover, estimate (3.10) implies the estimate

$$
\|T(t, h)\| \leq \sum_{k=0}^{\infty}\left\|T_{k}(t, h)\right\| \leq M e^{a t} \sum_{k=0}^{\infty} \frac{\left(M \sqrt{2}\|B\||h|_{L^{2}(\mathbb{R})} \sqrt{t}\right)^{k}}{\sqrt{k !}} \times \frac{1}{\sqrt{2^{k}}}
$$




$$
\leq M e^{a t}\left(\sum_{k=0}^{\infty} \frac{\left(2 M^{2}\|B\|^{2}|h|_{L^{2}(\mathbb{R})}^{2} t\right)^{k}}{k !}\right)^{1 / 2}\left(\sum_{k=0}^{\infty} \frac{1}{2^{k}}\right)^{1 / 2}=M \sqrt{2} e^{a t} \exp \left(M^{2}\|B\|^{2}|h|_{L^{2}(\mathbb{R})}^{2} t\right) .
$$

By (2.8), we have $\|\hat{\Phi}(h)\| \leq\|\Phi\|_{H S, p, 0} \exp \left(|h|_{p}^{2}\right)$ for $h \in \mathcal{S}$ and some $p \in \mathbb{N}$. Hence, for $t \geq 0$,

$$
\begin{gathered}
\|\hat{X}(t, h)\| \leq M \sqrt{2} e^{a t} \exp \left(M^{2}\|B\|^{2}|h|_{L^{2}(\mathbb{R})}^{2} t+|h|_{p}^{2}\right)\|\Phi\|_{H S, p, 0} \\
\leq M \sqrt{2} e^{a t} \exp \left(\left(M^{2}\|B\|^{2} t+1\right)|h|_{p}^{2}\right)\|\Phi\|_{H S, p, 0}, \quad h \in \mathcal{S} .
\end{gathered}
$$

Therefore, for $t \geq 0, \hat{X}(t, h)$ is the $S$-transform of only one generalized random variable $X(t)$ from the space $(\mathcal{S})_{-0}(H)$, and this variable is the unique solution of problem (3.2).

Corollary 2. Under the assumption of Theorem 2, for any $T>0$, there exist $q \in \mathbb{N}$ and $K>0$ such that, the solution $X(t)$ of Cauchy problem (3.2) satisfies the inequality

$$
\|X(t)\|_{\mathrm{HS}, q, 0} \leq K e^{a t}\|\Phi\|_{\mathrm{HS}, p, 0}, \quad 0 \leq t \leq T .
$$

Proof. As follows from Theorem 1 and estimate (3.14), for any $t \in[0 ; T]$ and any $q \in \mathbb{N}$ satisfying the condition

$$
2 e^{2}\left(M^{2}\|B\|^{2} T+1\right) \sum_{i=1}^{\infty}(2 i)^{-2(q-p)}<1
$$

the following estimate holds:

$$
\begin{gathered}
\|X(t)\|_{\mathrm{HS}, q, 0} \leq M \sqrt{2} e^{a t}\left(1-2 e^{2}\left(M^{2}\|B\|^{2} t+1\right) \sum_{i=1}^{\infty}(2 i)^{-2(q-p)}\right)^{-1 / 2}\|\Phi\|_{\mathrm{HS}, p, 0} \\
\leq M \sqrt{2} e^{a t}\left(1-2 e^{2}\left(M^{2}\|B\|^{2} T+1\right) \sum_{i=1}^{\infty}(2 i)^{-2(q-p)}\right)^{-1 / 2}\|\Phi\|_{\mathrm{HS}, p, 0} .
\end{gathered}
$$

Note that the length of the interval $[0 ; T]$ is defined by the choice of $q>p$, which is seen from inequality (3.16). The greater $q$, the longer the interval where estimate (3.15) is true.

Example 3. Let

$$
\begin{gathered}
H=L^{2}[0,1], \quad A=-\frac{d}{d s}+\mu(s), \quad \mu \in L_{\infty}[0,1] \\
\frac{d \mu}{d s} \in L_{\infty}[0,1], \quad \operatorname{dom} A=\left\{x \in L^{2}[0,1], \frac{d x}{d s} \in L^{2}[0,1], x(0)=0\right\} .
\end{gathered}
$$

The operator $A$ generates a semigroup of class $C_{0}$, which can be proved by methods from perturbation theory for semigroups: the operator $d \mu / d s$ generates a translation semigroup of class $C_{0}$ in the space $L^{2}[0,1]$, and the operator of multiplication by the function $\mu$ with the specified properties is bounded in $L^{2}[0,1]$; this perturbation of the operator preserves the property that the corresponding semigroup belongs to the class $C_{0}$.

Let $B(\cdot) \in \mathcal{L}(H ; \mathcal{L}(H))$ be defined by the equality

$$
[B(x) y](s):=\varepsilon \times x(s)(\psi * y)(s),
$$


where $\varepsilon>0$ and $\psi \in C_{0}^{\infty}(\mathbb{R})$ (an infinitely differentiable function with compact support). The operators $A$ and $B$ defined here satisfy the conditions of Theorem 2; hence, corresponding Cauchy problem (1.2) has a unique solution in $(\mathcal{S})_{-0}(H)$, and this solution is stable in the sense of $(3.15)$.

Equation with operators similar to $A$ arise, for example, in problems of population dynamics. Such an operator contains a term that generates a semigroup of translation type and describes the change in time of some numerical characteristic of a population (for example, the age of individuals or their size); it also contains terms that are operators of multiplication by a function and describe structural changes of the population related to such phenomena as the death or reproduction of individuals. For an appropriate choice of the parameter $\varepsilon$ and function $\psi$, the operator $B$ of form (3.17) can be considered as a stochastic perturbation of the operator of multiplication by the function $\mu$ (see, for example, [7], which studied an equation describing the evolution of a population of cells structured by size, which was stochastically perturbed in a similar way).

\section{CONCLUSIONS}

The introduction of a multiplicative stochastic perturbation (multiplicative noise) into a differential operator equation in a Hilbert space is an important problem with respect to both formulation and solution. The spaces $(\mathcal{S})_{-\rho}(H)$ of Hilbert-valued random variables introduced in the present paper made it possible, on the one hand, to consider a differential operator equation with cylindrical white noise and, on the other hand, to use the technique of reducing a Cauchy problem for a stochastic differential equation by means of the $S$-transformation to a Cauchy problem for a deterministic equation with further proof of the existence, uniqueness, and stability of its solutions in the space $(\mathcal{S})_{-0}(H)$.

\section{ACKNOWLEDGMENTS}

This work was supported by the Program of the Ministry of Education and Science of the Russian Federation (project no. 2.1.1/14118) and by the Russian Foundation for Basic Research (project no. 10-01-96003r).

\section{REFERENCES}

1. G. Da Prato and J. Zabczyk, Stochastic Equations in Infinite Dimensions (Cambridge Univ. Press, Cambridge, 1992), Ser. Encyclopedia of Mathematics and Its Applications, Vol. 44.

2. H.-H. Kuo, White Noise Distribution Theory (CRC, Boca Raton, 1996).

3. H. Holden, B. Øksendal, J. Ubøe, and T. Zhang, Stochastic Partial Differential Equations: A Modeling, White Noise Functional Approach (Birkhäuser, Boston, 1996).

4. A. Filinkov and J. Sorensen, Stoch. Stoch. Rep. 72 (3-4), 129 (2002).

5. I. V. Melnikova, A. I. Filinkov, and M. A. Alshansky, J. Math. Sci. (N. Y.) 116 (5), 3620 (2003).

6. M. A. Al'shanskii and I. V. Mel'nikova, Sb. Math. 202 (11-12), 1565 (2011).

7. M. A. Al'shanskii, in Mathematical Modeling and Boundary Value Problems: Proc. 7th All-Russian Sci. Conf., Part 3: Differential Equations and Boundary-Value Problems (Samarsk. Gos. Univ., Samara, 2010), pp. 15-18 [in Russian].

Translated by E. Vasil'eva 